\title{
THE ROLE OF MAPKS IN ADIPOCYTE DIFFERENTIATION AND OBESITY
}

Bost F.*, Aouadi M., Caron L. \& Binétruy B.*

INSERM U568, Université de Nice Sophia-Antipolis, Faculté de Médecine, Avenue de Valombrose, 06107 Nice cedex, France

Key words: MAP kinases; obesity; adipocyte differentiation; ERK; JNK; p38 MAPK

*To whom correspondence should be addressed:

Frédéric Bost or Bernard Binétruy

tel.: (33) 4933777 41, fax: (33) 493377701

bost@unice.fr

binetruy@unice.fr 


\section{ABSTRACT}

The ERK, p38 and JNK Mitogen Activated Protein Kinases (MAPKs) are intracellular signalling pathways that play a pivotal role in many essential cellular processes such as proliferation and differentiation. MAPKs are activated by a large variety of stimuli and one of their major functions is to connect cell surface receptors to transcription factors in the nucleus, which consequently triggers long-term cellular responses. This review focuses on their in vitro and in vivo roles in adipocyte differentiation and obesity. Hyperplasia of adipose tissue is a critical event for the development of obesity. Several studies have analysed the role of MAPKs in vitro in adipocyte differentiation of preadipocyte established cell lines. In the case of ERK, although the first data appeared contradictory, a consensus scenario arises: ERK would be necessary to initiate the preadipocyte into the differentiation process and, thereafter, this signal transduction pathway needs to be shut-off to proceed with adipocyte maturation. The limitation of these cellular models is that only terminal adipocyte differentiation can be analysed, eluding the early proliferative steps of adipogenesis. New insights are now emerging by investigations conducted either in vitro with the use of embryonic stem (ES) cells or in vivo with mice where these genes are invalidated. These studies not only confirm and/or precise the various functions of MAPKs in adipogenesis but, importantly, reveal unsuspected roles, for example JNK in obesity or ERK in adipogenesis of ES cells, and, for a given pathway, assign specific functions to each isoform. It appears now that a fine tuning of the MAPKs regulates both normal and pathological adipogenesis. The precise understanding of the cascade of these molecular events and the way to regulate them will be certainly crucial in order to efficiently fight obesity. 
Cells respond to extracellular signals by engaging a variety of intracellular signalling pathways, which trigger both immediate and long-term cell responses. While immediate responses involve mainly cytoplasmic events, long-term responses, by affecting gene expression, necessitate that cascades signal to the nucleus. The signalling pathways leading to activation of mitogen-activated protein kinases (MAPKs) represent a paradigm in cellular signalling research (see for an extensive review [1]). These pathways have the capacities to engage upon their activation both specific and distinct cascades from the cell membrane to the nucleus leading to gene regulation and long-term responses. Most of the knowledge comes from the study of three groups of MAPKs: extracellular signal-regulated kinases (ERKs) 1 and 2; c-Jun amino-terminal kinases (JNKs) 1, 2 and 3; and p38 MAPK (p38) $\alpha, \beta, \gamma$, and $\delta$. These kinases are serine/threonine kinases regulated by phosphorylation cascades organized in specific modules. All modules comprise two additional protein kinases activated in series and leading to activation of a specific MAP Kinase: a MAP Kinase Kinase (MAPKK), represented by MEK or MKK proteins, which phosphorylates a specific MAPK, and a MAP Kinase Kinase Kinase (MAPKKK), represented by Raf and MEKK proteins, which phosphorylates a specific MAPKK (Figure 1). The components of the different pathways upstream of MAPKKK are many and diverse but the best defined are the one used by the ERK pathway. Stimulation by appropriated ligands induces a signalling cascade linking membrane-associated tyrosine-kinase receptors to ERK proteins that implicates the Ras protein. The signalling from the membrane leading to activation of the p38 and JNK pathways is much less precisely defined and involves specific components such as Rac and cdc42 small GTPases. Besides the activities of the different components of the cascades themselves, there are two others important means to regulate specifically these signalling pathways: interfering with the scaffolding proteins and interfering with the MAPK phosphatases, specific for each pathway. 
Taking advantage of the development of specific chemical inhibitors for each MAPK pathway, numerous works have studied their biological functions, showing their involvement in a wide variety of cellular functions. These multiple functions are depending on the pathway that is activated and on the cellular model analysed. In addition, the duration of the stimulus can also affect the cellular response. A wide panel of different stimuli are capable to activate the MAPK pathways, but a good correlation has been found between the types of stimulus and the function assigned to the pathway. Schematically, ERK is preferentially activated by mitogens such as the serum or growth factors and, accordingly, this pathway is an important regulator of cell cycle and cell proliferation. p38 and JNK are more responsive to various stress stimuli from UV to cytokines and they have been involved in apoptosis and/or in the response to cellular stresses. Because they have been extensively studied (see for reviews [2] [3]), these general aspects will not be detailed in this review, except for their specific relevance towards adipogenesis.

Regarding the process of differentiation, the role of MAPKs is extremely complex and depends on multiple parameters. The complexity is due, firstly, to the biological process itself, which, most of the time, involves different successive steps. Furthermore, each of these steps can be modulated by MAPKs leading, sometimes, to opposite effects. Probably because this complexity renders experimental models extremely sensitive, most of the tools used for these studies have found their limitations. For example, in function of their concentration, it is known that chemical inhibitors of a given pathway differ in their inhibitory capacity and differ also in their properties to cross-talk with other patways. Therefore, interfering with a given pathway with chemical inhibitors can induce different biological effects. Alternatively, investigators have constructed cell lines, from various cellular models, overexpressing dominant-negative or activated forms of the genes encoding the components of MAPK pathways. While very informative, these experiments are not totally conclusive because of the complexity of the differentiation process and the possible cross-talk between the different 
pathways in such conditions. Finally, the knock-out of several components of MAPK pathways are now available, giving important information concerning their biological functions (see below). Altogether these different approaches draw a contrasting picture of the role of MAPKs in differentiation and demonstrate that they can modulate very efficiently this process.

Because of its essential role in cell proliferation and the fact that adipogenic stimuli, such as insulin, activate the ERK pathway, the role of this pathway in normal and pathological adipogenesis has been intensively investigated the last decade. Indeed, obesity is due to the hypertrophy of adipocytes and to the recruitment of new adipocytes from precursor cells, two processes largely dependent on regulation of adipocyte differentiation. In addition, obesity is associated with insulin resistance both in experimental models and in humans, and the role of adipose tissue and, consequently, of adipocyte differentiation is important in this pathology. Although the implication of MAPKs in insulin resistance is largely documented, our review does not discuss this aspect in details because Gual et al. review it in this series.

Although much of the knowledge originates from analysis of preadipocyte cell lines, such as 3T3-L1 or 3T3-F442A, various cellular models are now available; they are summarized in figure 2.

\section{The ERK pathway}

Whereas apparent contradictory results were first related in 3T3-L1 preadipocyte cells, recent results lead to the conclusion that the function of ERK in adipogenesis is multiple, and finally, by assigning distinct biological functions to each ERK isoforms, the analysis of mice lacking ERK1 or ERK2 has added a supplementary degree of complexity.

\section{The ERK pathway in adipogenesis: the model of preadipocyte cell lines}

The first paper demonstrating a potential link between ERK and adipocyte differentiation was published in 1991 [4]. The authors showed that transfection of 3T3-L1 
preadipocyte cells by an activated Ras expression vector led to growth arrest and terminal adipocyte differentiation. Since the oncogenic form of Ras protein is a strong activator of the ERK pathway, these results suggest a positive role for ERK in adipogenesis. More directly, using an oligonucleotide anti-sense strategy against ERK1 and 2, Sale and coworkers [5] demonstrated that the ERKs are required for differentiation of 3T3-L1 fibroblasts to adipocytes. This simple picture was then blurred by works demonstrating that the adipocytespecific transcription factor PPAR $\gamma$ is a substrate of ERK and that this phosphorylation decreases its transcriptional activity and inhibits adipocyte differentiation [6, 7]. Owing to the known role of ERK in cell proliferation, one could reconcile these contradictory results by hypothesizing that the function of ERK in adipogenesis has to be timely regulated: early on, ERK has to be turn on for a proliferative step, while latter on, it has to be shut-off to avoid PPAR $\gamma$ phospshorylation. This hypothesis is in agreement with the fact that the adipocyte differentiation process of 3T3-L1 requires, at the beginning, a precise proliferative step (called mitotic clonal expansion, MCE), which takes place post-confluency. MCE is initiated by adipogenic stimuli, such as insulin, which are known to activate the ERK pathway. Furthermore, PPAR $\gamma$ expression is not detected during MCE, and progressively increases during terminal differentiation, when ERK activity is returned to a low level. The prediction of such model is that inhibition of the ERK pathway, precisely during the MCE, would block adipocyte differentiation. This has been verified by the work of Tang et al. [8]. The authors showed that the specific and potent MEK-inhibitor U0126, administered during the MCE, efficiently blocks both ERK activity and adipocyte differentiation. Noteworthy, in this work, they also showed that PD98059, another MEK inhibitor, is less efficient in blocking ERK and, consequently, differentiation is not affected. These results can explain why, in previous works, PD98059 did not affect adipocyte differentiation $[9,10]$. Therefore, during MCE, a complete inhibition of ERK pathway seems to be required to see the effect on differentiation. Other works found a positive role for ERK in adipocyte differentiation, either in 3T3-L1 cells 
[11] or in other cellular models [12, 13], showing, in addition, that ERK activity is necessary for the expression of the crucial adipogenic regulators $\mathrm{C} / \mathrm{EBP} \alpha, \beta$ and $\delta$ and PPAR $\gamma$.

If ERK activation during the early proliferative step of differentiation is essential, the subsequent downregulation of this pathway seems also very important. This inhibition could be mediated by the modulation of two proteins: AEBP-1, which binds to ERK proteins and protects them from specific phosphatases, and the phosphatase MKP-1, which inactivates ERK proteins. Indeed, AEBP-1 expression is downregulated in mature adipocytes [14], and, conversely, MKP-1 expression is augmented [15].

\section{The ERK pathway in adipogenesis: the model of Embryonic Stem cells}

While the vast majority of studies have been performed in preadipocyte cell lines, the possibility to derive mature adipocytes directly from mouse Embryonic Stem (ES) cells has been described [16]. ES cells are pluripotent cells that differentiate into multiple cell lineages. At the opposite of preadipocyte cell lines that allow investigation of the terminal differentiation only, ES cells to adipocytes recapitulate the whole adipogenesis, giving access to the early steps of adipocyte differentiation. Adipocyte differentiation of ES cells can be achieved after a three weeks period [16] including a critical early treatment with retinoic acid followed by the application of classical adipogenic inducers. Under these conditions large clusters of mature adipocytes are present in 70 to $80 \%$ of the embryoid bodies. Our laboratory has investigated the role of the ERK pathway during ES cell commitment into the adipocyte lineage [17]. We showed that retinoic acid activates the ERK pathway, and not the other MAPK pathways, in embryoid bodies during early events of adipocyte differentiation. Inhibition of ERK activation using PD98059 or U0126 during this period results in a strong inhibition of adipocyte formation. By contrast, neurogenesis and myogenesis is not affected by PD98059. Our results strongly suggest an essential and specific role for ERK pathway in the early proliferative stage of adipogenesis; thereby, extending the role of this pathway throughout adipogenesis.

\section{Lessons from the ERK1 and ERK2 knockout mice}


Owing to the in vitro biological functions demonstrated for the ERK pathway, one could expect that knock-out (KO) of the corresponding genes would have pronounced phenotypes, particularly regarding adipogenesis. Interestingly, while invalidation of ERK2 isoform is embryonic lethal [18], ERK1 ${ }^{-/-}$mice are viable and fertile [19], showing that ERK1 and ERK2 have distinct biological functions. ERK1 deficient mice have defective thymocyte maturation [19] and enhanced long-term memory [20]. Our laboratory investigated the role of this isoform in adipocyte differentiation in vitro and in vivo by analysing adipogenesis in ERK $1^{-/-}$ mice. Compared to wild type control cells, Mouse Embryo Fibroblasts (MEF) and cultures of adult preadipocytes isolated from ERK1 ${ }^{-/}$animals exhibit impaired adipogenesis [21]. An inhibitor of the ERK pathway does not affect the residual adipogenesis of the ERK1 ${ }^{-/-}$cells, suggesting that ERK2 is not implicated in adipocyte differentiation. Importantly, mice lacking ERK1 have decreased adiposity and less adipocyte than wild type and ERK1 ${ }^{-/-}$mice challenged with high fat diet are resistant to obesity. In addition, ERK1 ${ }^{-/-}$mice are protected from insulin resistance and ERK activity is augmented in the adipose tissue of high fat diet fed wt animals, suggesting that ERK1 would be also implicated in insulin resistance. This is in agreement with data in humans where an increased ERK activity was observed in adipocytes of type 2 diabetes patients [22]. Altogether, our results clearly link ERK1 to the regulation of adipocyte differentiation, adiposity and high-fat diet induced obesity. This suggests that a therapeutic approach of obesity targeting specifically the ERK1 isoform and not ERK2 would be of particular interest.

\section{Molecular mechanisms linking the ERK pathway to adipogenesis}

One important question is left open by these studies: what are the molecular mechanisms underlying these various effects? is it a common mechanism or is each effect due to a specific ERK target? No precise answer to these questions can be given today but interesting tracks can be followed. Important adipogenic determinants have already been described as ERK targets: for example, ERK phosphorylation of C/EBP $\beta$ activates its 
transcriptional activity $[23,24]$ and $\mathrm{KO}$ of this gene leads to defective adipocyte differentiation [25]. Therefore, C/EBP $\beta$ represents a target that could account for the ERK effect on both 3T3-L1 adipocyte differentiation and the phenotype of ERK1 $1^{-/-}$mice. However this hypothesis is contradictory to recent results showing that $\mathrm{C} / \mathrm{EBP} \beta$ is a preferential target of ERK2 and not ERK1 [26]. Further works are necessary to answer this question; for example, it would be interesting to investigate the effect of an in vivo gene replacement of wt C/EBP $\beta$ by a mutated C/EBP $\beta$ on the ERK phosphorylation site.

Regarding the increased insulin sensitivity displayed by ERK1 $1^{-/-}$mice, one interesting potential ERK target is the IRS1 (Insulin Receptor Substrate 1) protein, a downstream effector of the insulin receptor. Indeed IRS1 protein is phosphorylated by the ERK pathway and this serine phosphorylation exerts an inhibitory effect on the insulin signalling, contributing to the insulin resistance state associated to obesity (see [27] and Gual et al., in this series). Whether IRS1 is a specific target of ERK1 needs to be determined.

\section{The p38MAPK pathway}

As for the ERK pathway opposing roles are described for p38 in adipocyte differentiation. Most studies describing a role of p38MAPK were performed in 3T3-L1 preadipocyte cell lines and used pyridinyl imidazole inhibitors such as SB203580 to inhibit p38 activation. Engelman et al. were the first to describe a positive role for p38 in adipogenesis [28, 29]. They showed that addition of the p38 inhibitors early in 3T3-L1 differentiation decreased adipocyte formation. They attributed this inhibition to a decrease in C/EBP $\beta$ phosphorylation and its post-translational activation. Furthermore, they showed that overexpression of MKK6(Glu), a constitutively active form of MKK6 (upstream activator of p38), during 96 hours is sufficient to stimulate 3T3-L1 differentiation into adipocyte. However, prolonged expression of MKK6(Glu) resulted in the death of the adipocytes, showing that activation of p38 can have different effects depending on the stage of 
differentiation. More recently, Hata et al. [30] demonstrated that Bone Morphogenic Protein-2 (BMP-2) oriented pluripotent mesenchymal cell lines C3H10T1/2 into the adipocyte lineage through activation of p38. Using p38 chemical inhibitors the authors observed a decrease in BMP-2-induced adipocyte formation and associated this defect to a decrease in PPAR $\gamma$ transactivation activity. Other reports performed in 3T3-L1 with SB203580 confirmed that p38 activation is required for adipocyte differentiation in these cell lines [31]. Yet, in human preadipocytes treated with p38 inhibitors, only a moderate decrease in adipogenesis was observed [32]. Other reports show opposite results and demonstrate that p38 activity inhibits adipocyte differentiation. For example, Wang et al. showed that CHOP (GAD153), a dominant-negative regulator of C/EBP, is phosphorylated in vitro and in vivo by p38 on Ser78 and Ser81 [33]. Interestingly, overexpression of wt CHOP blocks adipogenic differentiation of 3T3-L1 [34], whereas the non-phosphorylatable form of $\mathrm{CHOP}\left(\mathrm{CHOPAla}^{78,81}\right)$ is a poor inhibitor of adipogenesis. The authors conclude that CHOP phosphorylation by p38 is required for its full inhibitory effect, suggesting that p38 activity blocks adipocyte differentiation. A similar conclusion was made for the transcription factor NFATc4 (Nuclear factor of activated $\mathrm{T}$ cells) [35], which is implicated in adipocyte differentiation [36]. Dephosphorylation of NFAT promotes its nuclear localization. Once dephosphorylated and translocated into the nucleus, NFAT4c increases PPAR $\gamma$ expression and therefore promotes adipogenesis. Yang et al. showed that NFATc4 is phosphorylated by p38 and this phosphorylation inhibits adipose cell formation in NIH3T3. In order to have a clear picture of the implication of p38 in adipogenesis, studies should be performed in various cell models using not only inhibitors but also genetic approaches. Invalidation of p38 isoforms would be of great interest. Unfortunately, only the $\mathrm{p} 38 \alpha^{-/-}$mice are available so far and the animals die in utero between day 11.5 and 12.5 [37]. The characterization and study of tissue-specific knock-out of the different p38 isoforms would certainly help to define the role of this pathway. 
Type 2 diabetes is frequently associated with obesity and is characterized by insulin resistance. Several recent studies have shown that inhibition of p38 improves glucose transport in L6 and 3T3L1 [38, 39]. These results correlate with the study of Carlson et al. showing that p38 activity participates in a downregulation of GLUT4 expression and is significantly higher in isolated adipocytes from type-2 diabetes patients [22]. However, further studies are necessary to define the mechanisms involved with the activation of p38 and insulin-resistance, as well as the connection between p38 activation and regulation of GLUT4 expression. Genetically modified animals would also be of interest to study the role of this pathway in insulin-resistance.

\section{The JNK pathway}

Two recent papers showed that JNK1 and the scaffold protein JIP1 (JNK-interacting protein 1) are implicated in the development of obesity. The invalidation of JNK1, but not JNK2, leads to the resistance to high-fat diet induced obesity. Furthermore, ob/ob-JNK1 animals are also resistant to obesity [40]. Similarly, JIP1 deficiency provides partial resistance to obesity [41]. Serine phosphorylation of IRS-1 (Insulin receptor substrate 1) is known to participate in insulin resistance (see Gual et al., in this series, [27]). In both studies, the absence of JNK1 and JIP1 lead to a better insulin sensitivity attributed to a reduced phosphorylation of IRS-1 on Ser307. Finally, if it is clear that the JNK pathway is involved in insulin signalling there is no evidence for a role of this pathway in adipocyte differentiation.

\section{Conclusion}

MAPK pathways are able to regulate adipogenesis at each steps of the process, from stem cells to adipocytes (the various effects are summarised in Figure 3). While the ERK pathway is involved throughout adipogenesis, displaying both positive and negative effects, p38 and JNK seem to have more restricted potentials. Interestingly, some of these effects 
overlap each other's: ERK and p38 are required during the MCE of preadipocytes and ERK1 and JNK1 KOs both lead to resistance to obesity and hypersensitivity to insulin. Whether these observations correspond to common or distinct molecular mechanisms need to be determined.

Because of the pleiotropic functions of MAPK pathways, the precise understanding of these mechanisms will certainly be crucial in order to develop potent and specific drugs against obesity modulating the MAPK pathways. Furthermore, targeting specific isoforms, for example ERK1 or JNK1, without affecting the others, seems to be a promising approach to avoid interference with other essential MAPK functions.

\section{Acknowledgements}

We thank Y. Le Marchand-Brustel and J.F. Tanti for critical reading of the manuscript, the Fondation Bettencourt-Schueller and the Region Provence Alpes Côte-d'Azur for support. 


\section{REFERENCES}

[1] G. Pearson, F. Robinson, T. Beers Gibson, B. E. Xu, M. Karandikar, K. Berman and M. H. Cobb, Mitogen-activated protein (MAP) kinase pathways: regulation and physiological functions, Endocr Rev 22 (2001) 153-83.

[2] F. Chang, L. S. Steelman, J. G. Shelton, J. T. Lee, P. M. Navolanic, W. L. Blalock, R. Franklin and J. A. McCubrey, Regulation of cell cycle progression and apoptosis by the Ras/Raf/MEK/ERK pathway (Review), Int J Oncol 22 (2003) 469-80.

[3] A. M. Manning and R. J. Davis, Targeting JNK for therapeutic benefit: from junk to gold ?, Nat Rev Drug Discov 2 (2003) 554-65.

[4] M. Benito, A. Porras, A. R. Nebreda and E. Santos, Differentiation of 3T3-L1 fibroblasts to adipocytes induced by transfection of ras oncogenes, Science 253 (1991) 565-8.

[5] E. M. Sale, P. G. Atkinson and G. J. Sale, Requirement of MAP kinase for differentiation of fibroblasts to adipocytes, for insulin activation of p90 S6 kinase and for insulin or serum stimulation of DNA synthesis, Embo J 14 (1995) 674-84.

[6] H. S. Camp and S. R. Tafuri, Regulation of peroxisome proliferator-activated receptor gamma activity by mitogen-activated protein kinase, J Biol Chem 272 (1997) 10811-6.

[7] E. Hu, J. B. Kim, P. Sarraf and B. M. Spiegelman, Inhibition of adipogenesis through MAP kinase-mediated phosphorylation of PPARgamma, Science 274 (1996) 2100-3.

[8] Q. Q. Tang, T. C. Otto and M. D. Lane, Mitotic clonal expansion: a synchronous process required for adipogenesis, Proc Natl Acad Sci U S A 100 (2003) 44-9. 
[9] J. Font de Mora, A. Porras, N. Ahn and E. Santos, Mitogen-activated protein kinase activation is not necessary for, but antagonizes, 3T3-L1 adipocytic differentiation, Mol Cell Biol 17 (1997) 6068-75.

[10] Z. Qiu, Y. Wei, N. Chen, M. Jiang, J. Wu and K. Liao, DNA synthesis and mitotic clonal expansion is not a required step for $3 \mathrm{~T} 3-\mathrm{L} 1$ preadipocyte differentiation into adipocytes, J Biol Chem 276 (2001) 11988-95.

[11] D. Prusty, B. H. Park, K. E. Davis and S. R. Farmer, Activation of MEK/ERK signaling promotes adipogenesis by enhancing peroxisome proliferator-activated receptor gamma (PPARgamma ) and C/EBPalpha gene expression during the differentiation of 3T3-L1 preadipocytes, J Biol Chem 277 (2002) 46226-32. 2002.

[12] J. Aubert, S. Dessolin, N. Belmonte, M. Li, F. R. McKenzie, L. Staccini, P. Villageois, B. Barhanin, A. Vernallis, A. G. Smith, G. Ailhaud and C. Dani, Leukemia inhibitory factor and its receptor promote adipocyte differentiation via the mitogen-activated protein kinase cascade, J Biol Chem 274 (1999) 24965-72.

[13] N. Belmonte, B. W. Phillips, F. Massiera, P. Villageois, B. Wdziekonski, P. SaintMarc, J. Nichols, J. Aubert, K. Saeki, A. Yuo, S. Narumiya, G. Ailhaud and C. Dani, Activation of extracellular signal-regulated kinases and CREB/ATF-1 mediate the expression of CCAAT/enhancer binding proteins beta and -delta in preadipocytes, Mol Endocrinol 15 (2001) 2037-49.

[14] S. W. Kim, A. M. Muise, P. J. Lyons and H. S. Ro, Regulation of adipogenesis by a transcriptional repressor that modulates MAPK activation, J Biol Chem 276 (2001) 10199-206.

[15] H. Sakaue, W. Ogawa, T. Nakamura, T. Mori, K. Nakamura and M. Kasuga, Role of MAPK Phosphatase-1 (MKP-1) in Adipocyte Differentiation, J Biol Chem 279 (2004) 39951-7. 
[16] C. Dani, A. G. Smith, S. Dessolin, P. Leroy, L. Staccini, P. Villageois, C. Darimont and G. Ailhaud, Differentiation of embryonic stem cells into adipocytes in vitro, J Cell Sci 110 (1997) 1279-85.

[17] F. Bost, L. Caron, I. Marchetti, C. Dani, Y. Le Marchand-Brustel and B. Binetruy, Retinoic acid activation of the ERK pathway is required for embryonic stem cell commitment into the adipocyte lineage, Biochem J 361 (2002) 621-7.

[18] M. K. Saba-El-Leil, F. D. Vella, B. Vernay, L. Voisin, L. Chen, N. Labrecque, S. L. Ang and S. Meloche, An essential function of the mitogen-activated protein kinase Erk2 in mouse trophoblast development, EMBO Rep 4 (2003) 964-8.

[19] G. Pages, S. Guerin, D. Grall, F. Bonino, A. Smith, F. Anjuere, P. Auberger and J. Pouyssegur, Defective thymocyte maturation in p44 MAP kinase (Erk 1) knockout mice, Science 286 (1999) 1374-7.

[20] C. Mazzucchelli, C. Vantaggiato, A. Ciamei, S. Fasano, P. Pakhotin, W. Krezel, H. Welzl, D. P. Wolfer, G. Pages, O. Valverde, A. Marowsky, A. Porrazzo, P. C. Orban, R. Maldonado, M. U. Ehrengruber, V. Cestari, H. P. Lipp, P. F. Chapman, J. Pouyssegur and R. Brambilla, Knockout of ERK1 MAP kinase enhances synaptic plasticity in the striatum and facilitates striatal-mediated learning and memory, Neuron 34 (2002) 807-20.

[21] F. Bost, M. Aouadi, L. Caron, P. C. Even, N. Belmonte, M. Prot, C. Dani, P. Hofman, G. Pages, J. Pouyssegur, Y. Le Marchand and B. Binetruy, The erk1 isoform is specifically required for in vitro and in vivo adipogenesis, Diabetes in press.

[22] C. J. Carlson, S. Koterski, R. J. Sciotti, G. B. Poccard and C. M. Rondinone, Enhanced basal activation of mitogen-activated protein kinases in adipocytes from type 2 diabetes: potential role of p38 in the downregulation of GLUT4 expression, Diabetes 52 (2003) 634-41. 
[23] J. Hu, S. K. Roy, P. S. Shapiro, S. R. Rodig, S. P. Reddy, L. C. Platanias, R. D. Schreiber and D. V. Kalvakolanu, ERK1 and ERK2 activate CCAAAT/enhancer-binding protein-beta-dependent gene transcription in response to interferon-gamma, J Biol Chem 276 (2001) 287-97.

[24] C. Trautwein, C. Caelles, P. van der Geer, T. Hunter, M. Karin and M. Chojkier, Transactivation by NF-IL6/LAP is enhanced by phosphorylation of its activation domain, Nature 364 (1993) 544-7.

[25] T. Tanaka, N. Yoshida, T. Kishimoto and S. Akira, Defective adipocyte differentiation in mice lacking the C/EBPbeta and/or C/EBPdelta gene, Embo J 16 (1997) 7432-43.

[26] M. Hanlon, T. W. Sturgill and L. Sealy, ERK2- and p90(Rsk2)-dependent pathways regulate the CCAAT/enhancer-binding protein-beta interaction with serum response factor, J Biol Chem 276 (2001) 38449-56.

[27] Y. Zick, Insulin resistance: a phosphorylation-based uncoupling of insulin signaling, Trends Cell Biol 11 (2001) 437-41.

[28] J. A. Engelman, M. P. Lisanti and P. E. Scherer, Specific inhibitors of p38 mitogen-activated protein kinase block 3T3-L1 adipogenesis, J Biol Chem 273 (1998) 32111-20.

[29] J. A. Engelman, A. H. Berg, R. Y. Lewis, A. Lin, M. P. Lisanti and P. E. Scherer, Constitutively active mitogen-activated protein kinase kinase 6 (MKK6) or salicylate induces spontaneous 3T3-L1 adipogenesis, J Biol Chem 274 (1999) 35630-8.

[30] K. Hata, R. Nishimura, F. Ikeda, K. Yamashita, T. Matsubara, T. Nokubi and T. Yoneda, Differential roles of Smad1 and p38 kinase in regulation of peroxisome proliferator-activating receptor gamma during bone morphogenetic protein 2induced adipogenesis, Mol Biol Cell 14 (2003) 545-55. 
[31] T. Takenouchi, Y. Takayama and T. Takezawa, Co-treatment with dexamethasone and octanoate induces adipogenesis in 3T3-L1 cells, Cell Biol Int 28 (2004) 209-16.

[32] N. G. Patel, J. C. Holder, S. A. Smith, S. Kumar and M. C. Eggo, Differential regulation of lipogenesis and leptin production by independent signaling pathways and rosiglitazone during human adipocyte differentiation, Diabetes 52 (2003) 43-50.

[33] X. Z. Wang and D. Ron, Stress-induced phosphorylation and activation of the transcription factor CHOP (GADD153) by p38 MAP Kinase, Science 272 (1996) 1347-9.

[34] N. Batchvarova, X. Z. Wang and D. Ron, Inhibition of adipogenesis by the stressinduced protein CHOP (Gadd153), Embo J 14 (1995) 4654-61.

[35] T. T. Yang, Q. Xiong, H. Enslen, R. J. Davis and C. W. Chow, Phosphorylation of NFATc4 by p38 mitogen-activated protein kinases, Mol Cell Biol 22 (2002) 3892904.

[36] I. C. Ho, J. H. Kim, J. W. Rooney, B. M. Spiegelman and L. H. Glimcher, A potential role for the nuclear factor of activated $T$ cells family of transcriptional regulatory proteins in adipogenesis, Proc Natl Acad Sci U S A 95 (1998) 1553741.

[37] K. Tamura, T. Sudo, U. Senftleben, A. M. Dadak, R. Johnson and M. Karin, Requirement for p38alpha in erythropoietin expression: a role for stress kinases in erythropoiesis, Cell 102 (2000) 221-31.

[38] L. M. Furtado, R. Somwar, G. Sweeney, W. Niu and A. Klip, Activation of the glucose transporter GLUT4 by insulin, Biochem Cell Biol 80 (2002) 569-78.

[39] L. Michelle Furtado, V. Poon and A. Klip, GLUT4 activation: thoughts on possible mechanisms, Acta Physiol Scand 178 (2003) 287-96. 
[40] J. Hirosumi, G. Tuncman, L. Chang, C. Z. Gorgun, K. T. Uysal, K. Maeda, M.

Karin and G. S. Hotamisligil, A central role for JNK in obesity and insulin resistance, Nature 420 (2002) 333-6.

[41] A. Jaeschke, M. Czech and R. Davis, An essaential role of the JIP1 scaffold protein for JNK activation in adipose tissue, Genes \& Dev 18 (2004) 1976-80.

\section{Legend to figures}

figure 1: MAPKs signal transduction pathways

figure 2: the different in vitro models to study adipocyte differentiation

The specific adipogenic inducers for each model are indicated: retinoic acid (RA); insulin and thyroid hormone (Ins+T3); insulin, 3-isobutyl-1-methylxanthine (IBMX) and dexamethasone (dex).

figure 3: involvement of the MAPKs at the various steps of adipogenesis 